\title{
Neuromarketing: un acercamiento sobre su influencia en las decisiones de compra*
}

\section{Neuromarketing: an approach on its influence on purchasing decisions}

Poveda Velasco Juan Carlos ${ }^{1}$

Recebido em: 04/09/2016. Aprovado em: 16/10/2016.

1 Universidad Mayor, Real y Pontificia de San Francisco Xavier de Chuquisaca - Espanha.

\section{Resumen}

El reporte tiene como objetivo identificar como las decisiones de compra son influenciadas por el neuromarketing además de hacer una relación de las condiciones que esta actividad propone en el campo del marketing. Se aborda un tema de actualidad que si bien no es nuevo, está vigente en diferentes espacios de discusión científica, académica y empresarial. El paradigma del Marketing sigue siendo el del Marketing Relacional, pero se puede percibir el rápido avance que actualmente presenta. El comportamiento del consumidor relacionado con las decisiones de compra es de especial interés para las empresas, sobre todo por la finitud de los compradores, lo que hace que exista una gran competencia por ocupar un lugar privilegiado en las preferencias de los consumidores. El neuromarketing es una técnica que busca mejorar el conocimiento de las preferencias y sus motivadores. Para el logro del objetivo la metodología fue exploratoria a partir de fuentes secundarias de publicaciones en español realizadas en el google académico en el periodo 2012 - 2016. Los resultados indican que el neuromarketing permite identificar, conocer y pronosticar las conductas que influyen en los comportamientos de las personas al momento de tomar una decisión de compra.

Palavras-chave: Neuromarketing. Decisiones de compra. Comportamiento del consumidor. Tecnología de marketing. Mercado de marketing.

\section{Abstract}

The report aims to identify how purchase decisions are influenced by neuromarketing; besides, it aims to present new conditions that this activity offers in the marketing field. This particular issue that while not new, is in force in different areas of scientific, academic and business discussion addresses. The paradigm of marketing is still Relationship Marketing, however, permanent change can be seen in the field of marketing. Consumer behavior related to purchasing decisions is of particular interest to businesses, especially in the finiteness of buyers, which means that there is great competition to occupy a privileged place in the preferences of consumers. Neuromarketing is a technique that seeks to improve knowledge of the preferences and motivators. To achieve the objective methodology was exploratory from secondary sources of publications in Spanish conducted in academic google in the period 2012 - 2016. The results indicate that neuromarketing can identify, understand and predict the behaviors that influence behavior people when making a purchase decision.

Keywords: Neuromarketing. Purchasing decisions. Consumer behavior. Neuromarketing technology. Neuromarketing market. 


\section{Introducción}

En el mundo actual con una alta competencia empresarial por conquistar la atención de los consumidores, surgen diversos enfoques investigativos para intentar comprender mejor el comportamiento de las personas que influye en sus decisiones de compra, una técnica novedosa es el neuromarketing.

Paul D. MacLean, según menciona Diez (2011), en 1949 propuso una teoría donde las estructuras neuronales en el cerebro determinan las emociones en el ser humano, el sistema límbico es el lugar donde se ubican estos procesos emocionales en todos los niveles de complejidad, a partir de esta teoría surge la hipótesis del cerebro triple; es a partir de esta teoría que surgen las neurociencias y a partir de la misma es que se desarrolla el neuromarketing.

Según Ospina Gonzalez (2014) el neuromarketing contribuye a medir las emociones, sentimientos, sensaciones de las personas como resultado de estímulos externos. A través del neuromarketing se pueden entender las reacciones y comportamientos de las personas a la comunicación publicitaria de las personas, permitiendo a las empresas, a través de la comprensión del comportamiento de las personas, maximizar sus beneficios y reducir los costes publicitarios. Según Canales Ronda (2013) el neuromarketing es una nueva forma de investigar el comportamiento del consumidor, de manera que se pueda explicar mejor las conductas de las personas al momento de tomar decisiones de compra.

Aparentemente, por lo descrito por diferentes autores, existe una relación entre las decisiones de compra y el neuromarketing, en ese contexto con la presente investigación se realizará una revisión de estudios e investigaciones publicadas en español en el google académico en el periodo 2012 a 2016 para intentar resolver el siguiente problema ¿cómo las decisiones de compra de las personas son influenciadas por el neuromarketing?

Una vez etablecido el problema de la investigación el objectivo de este trabajo es Identificar como las decisiones de compra son influenciadas por el neuromarketing.

\section{Metodología}

La investigación es de tipo exploratoria orientada al logro del objetivo a partir de la revisión de fuentes secundarias. tores conceptuales que explican el neuromarketing como una técnica complementaria y contributiva del marketing.

-Después se buscan en el internet fuentes secundarias que proporcionen indicios teóricos y empíricos que contribuyan a responder el problema planteado.

-Finalmente, se contrastan estos resultados para el logro del objetivo.

\section{El cerebro triple y la percepción sensorial cognitiva}

De acuerdo a Camacho Castellanos (2016) la evolución del cerebro ha implicado el desarrollo de tres niveles interconectados pero que funcionan y responden en áreas especializadas diferenciadas, cada uno de ellos es descrito de la siguiente manera:

El Cerebro reptiliano: Independiente de la voluntad y relacionado con las conductas básicas e instintivas de las personas; en esta parte se alojan las necesidades primarias de protección, reproducción, sobrevivencia, territorialidad, alimentación e incluso violencia. Sobre estas necesidades los seres humanos tienen muy poco control.

El Cerebro límbico: Es la responsable de las emociones, de la creatividad, de las sensaciones subconscientes. A esta parte se le atribuye emociones como el amor, el odio, la alegría, la melancolía. También se atribuye el desarrollo de las capacidades artísticas, la música, pintura, etc.

El Cerebro neo-córtex: La parte racional, que rige el comportamiento socialmente aceptable. Es la parte que limita las conductas básicas y las emociones. En este nivel es donde se articula el pensamiento y la comunicación del mismo, lo que también significa que es donde se determina el actuar racional y aceptado del ser humano.

En la interacción integrada de estos tres niveles del cerebro es donde se generan los comportamientos humanos, desde los más básicos como el comer para satisfacer el hambre, hasta otros más complejos como elegir el cubierto socialmente adecuado cuando se come en un banquete. Las emociones que conducen a las decisiones de compra para la satisfacción de una necesidad, no importa si esta es básica o más compleja, son el resultado de la interacción de estos niveles de cerebro.

Silva Bustillos (2015) en su estudio sobre Programación Neurolinguistica, Neuromarketing y Placebo, 
describe como la información que reciben las personas y la comprensión de esa información, involucra a dos áreas del cerébro humano, una primera que se encarga de la producción del habla y el procesamiento del lenguaje, que corresponde al área de Broca, mientras que la otra parte se encarga de decodificar y la comprensión de la información, denominada el área de Wernike; estableciendo así el sistema fisiológico de comunicación humana. Como parte de la explicación del sistema de comunicación humana, se indica también que es a través de la percepción sensorial que los datos recibidos por los diferentes sentidos y sistemas sensoriales son transformados en constructos mentales que dan valor y significado a esos datos.

Es a partir del sistema de comunicación humana y la percepción sensorial como las personas son capaces de crear un modelo interpretativo de su entorno, Silva Bustillos (2015) denomina arquitectura de modelo referencial (AMR) a este proceso, indicando además que las diferentes funciones del proceso de modelamiento tienen correlación biológica con la estructura cerebral y es ahí donde se generan las conductas humanas relacionadas con las decisiones de compra.

De acuerdo a lo propuesto en la AMR, el modelamiento del mundo, y en consecuencia, el comportamiento generado por una persona va a depender del juicio de valor otorgado a la percepción sensorial, la cual es filtrada por parte de la base de conocimientos propia del individuo. De acuerdo a lo anterior, la base de conocimientos de una persona incide directamente sobre la toma de decisiones y, en consecuencia debe ser la pieza clave a la hora de decidir sobre el consumo de bienes y servicios. (SILVA BUSTILLOS, 2015 , p.)

Entonces, las decisiones emocionales para satisfacer una necesidad, tendrían base en la estructura biológica del cerebro humano, en las percepciones sensoriales que estimulan diferentes áreas de esta estructura cerebral, en la base de conocimientos residentes en los niveles del cerebro triple, y la arquitectura del modelo referencial o modelo interpretativo del mundo, del ser humano.

\section{Necesidades como motivadores de} compra

Aceptando que la acción de compra es resultado de una decisión emocional para satisfacer una necesidad, es que se encuentra una relación entre las decisiones de compra y las necesidades; Abraham Maslow en 1943 en su obra "Una Teoría de la Motivación Humana" realizó una categorización de las necesidades humanas, sobre este aspecto Camacho Castellanos (2016), dice que como el marketing busca en ultima instancia satisfacer una necesidad con el mejor bien - producto o servicio - existe una relación entre las necesidades y las decisiones de compra, es más, propone que es ahí donde interviene el neuromarketing.

Camacho Castellanos (2016), resume las categorías de Maslow para las necesidades, de la siguiente manera:

\subsection{Necesidades Fisiológicas}

En este estadio se encuentran aquellas necesidades básicas para la subsistencia del ser humano y la satisfacción de los instintos requeridos para la procreación. El hambre, la sed, el sueño, la eliminación de residuos, el sexo y la homeostasis. Estas necesidades generalmente se asocian con los instintos, con el área del cerebro que se identifica como reptiliano.

\subsection{Necesidades de Seguridad}

En este nivel se encuentran las necesidades que representan protección y seguridad del individuo frente a su entorno. Vivienda, ropa, la protección de su derecho de propiedad, el contar con un ingreso económico (empleo o empresa) estable para solventar las necesidades inherentes a su ser y a la de aquellos que dependen de él. Estas necesidades presentan una carga de dos tipos de reacciones, específicamente los instintos y las emociones, por ello se encuentra entre el cerebro reptiliano y el límbico.

\subsection{Necesidades de Afiliación}

Estas necesidades corresponden a todo lo que implica la relación con nuestros semejantes, la amistad, el afecto, las relaciones de pareja y relaciones en el medio familiar. Estas necesidades son de un alto contenido emocional, por lo que se encuentra localizadas en el cerebro límbico.

\subsection{Necesidades de Reconocimiento}

Relacionadas con el ego del individuo, la individualidad, el reconocimiento de capacidades propias. Son necesidades basadas en en lograr el reconocimiento de aquellos que interactúan social, laboral y familiarmente con ellos. Estas se ubican entre el cerebro límbico y el neo-córtex. 


\subsection{Necesidades de Auto Reconocimiento}

En la cúspide de la pirámide de Maslow se encuentran estas necesidades, están relacionadas con el racioncinio, el conocimiento, la lógica, la ética, la moralidad, los valores, el sentido de justicia, la innovación y la capacidad de solucionar problemas. Corresponde al cerebo neo-córtex.

\section{Tecnologías de medición neurocientíficas}

Las neurociencias han desarrollado una serie de tecnologías para medir las diferentes reacciones de los seres humanos ante los estímulos externos e internos, en esa medida muchas de estas tecnologías también se están aplicando para medir las reacciones biológicas de las personas ante estímulos produccidos por ciertos bienes, en consecuencia midiendo los efectos del neuromarketing.

Según Martín Castejón y Lázaro Pérez (2013); Canales Ronda (2013), el neuromarketing hace uso de las mismas tecnologías que las neurociencias para medir la salud del cerebro, sólo que con una orientación hacia la respuesta de estímulos a partir de acciones de marketing; las tecnologías y medios usados en la actualidad por el neuromarketing son:

\subsection{Resonancia Magnética funcional (fMRI)}

La imagen por resonancia magnética funcional se utiliza para estudiar las funciones de las áreas del cerebro, y para conocer la forma en que funciona las mismas. Monitoriza las funciones fisiológicas pero, en este caso, de manera no invasiva, permite identificar como procesa la información el cerebro y como de acuerdo a ese procesamiento conduce al individuo a tomar determinadas decisiones. El fMRI es costosa pero genera resultados muy completos y confiables.

\subsection{Encefalografía (EEG)}

El electroencefalograma es un examen neurofisiológico que se hace para medir la actividad eléctrica en el cerebro. En presencia de estímulos audiovisuales, como por ejemplo los anuncios publicitarios, las neuronas producen cierta actividad eléctrica y esta herramienta amplifica esta información para un posterior análisis. El EEG es probablemente la técnica más barata y accesible, la más amigable para los sujetos estudiados.

\subsection{Magneto Encefalografía (MEG)}

Esta tecnología es otra técnica no invasiva que mide las fluctuaciones magnéticas que se producen en el cerebro como resultado de la actividad de las neuronas. La calidad de la señal y la resolución temporal de la MEG son superiores a los de la EEG, aunque no puede utilizarse si el sujeto de estudio tiene implantes metálicos.

- $\quad$ Eye tracking (ET)

- Identifica el movimiento de la córnea del ojo y la atención espacial del mismo.

- Análisis facial (FACS)

- Analiza los movimientos de los diferentes músculos de la cara relacionados con la expresión de emociones.

- Ritmo del corazón (HR)

- Mide los cambios en el ritmo cardiáco por efecto de estímulos externos.

- Test de asociaciones implícitas (IAT)

- Mide el tiempo de respuesta de la persona ante estímulos comerciales.

- Análisis de movimientos

- Identifica mediante acelerómetros las reacciones físicas ante estímulos diferentes.

- Ritmo de la respiración (RR)

- Analiza los cambios de frecuencia en la respiración atribuidas a emociones.

- Análisis de la piel (EDA-SCR, GSR)

Mediciones de respuesta del cerebro mediante mediciones de los niveles de sudoración como efecto de un estímulo.

- Tonos de la voz (VPA)

Analisa las variaciones de tonos y vibraciones de las cuerdas vocales como resultado de las variaciones de entorno y emocionales.

Todas estas tecnologías de medición están orientadas a examinar el comportamiento del cerebro y su efecto en el cuerpo del ser humano como respuesta a estímulos externos percibidos sensorialmente.

\section{Motivadores de las decisiones de compra}

Según Vidal Brazales (2015) existen varias propuestas para explicar el proceso de toma de deciones del comprador, pero las clasificaciones más frecuentes, a partir de los motivadores de la decisión de compra, son las siguientes:

- la económica, decisión reflexiva, donde se hace 
una evaluación racional de las ventajas y desventajas de las diferentes alternativas que se tienen.

- la pasiva, donde la publicidad influye en la decisión de compra del consumidor, quién responde de forma automática, impulsiva e irracional a esos estímulos.

- la cognitiva, basada en el conocimiento y diferenciación del producto, el consumidor identifica y busca los bienes - productos o servicios - que mejor satisfacen sus necesidades a partir del análisis de la información comunicada por las empresas. Influye también las experiencias pasadas.

- la emocional, estas decisiones están basadas en la satisfacción emocional de las necesidades, el consumidor se guía esencialmente por impulsos y emociones antes que por el pragmatismo de la compra.

Una decisión de compra no se basa exclusivamente en uno de estos motivadores, según Vidal Brazales (2015) el comportamiento del consumidor es afectado por todas las experiencias asociadas con el uso o el consumo de un bien, además de las sensaciones de placer que se derivan del mismo.

\section{Marketing y neuromarketing}

Según Tallada Casanova, (2015), citando a (MONGE, 2011; ARIEL, 2010), el marketing ayuda a las empresas a organizar el proceso de venta de un bien, a través de la generación de relaciones que permitan adecuar este bien a las necesidades del consumidor, de manera que existe interacción entre la empresa y el consumidor a partir de la interpretación de los hábitos expresados en deseos del consumidor.

Por otro lado, el neuromarketing aporta al marketing con la generación de información a partir de la comprensión con base biológica del comportamiento de las personas.

El neuromarketing influye en el proceso de decisión de compra porque permite medir la parte racional, emocional e instintiva de las personas para lograr creas estrategias de mercadeo efectivas que lleguen directamente al cerebro del consumidor despertando sus instintos, sus emociones y su racionalidad. Según la fase del ciclo en la que se encuentre el consumidor, estos tres factores influyen de diferente forma en el consumidor así como los estímulos externos que reciba. (OSPINA GONZALEZ, 2014, p. 52)
Una mejor comprensión del comportamiento del consumidor permite a las empresas ser más eficiente y eficaces en la implementación de estrategias de marketing, satisfaciendo mejor las necesidades de sus clientes.

Según Avendaño Castro (2013) el neuromarketing es una técnica del marketing que contribuye a la comprensión de los procesos cerebrales que determinan el comportamiento de las personas para la toma de decisiones de compra, es decir, como las personas perciben los estímulos externos y son influenciados para elegir un bien, entonces, a través del neuromarketing las empresas pueden conocer la respuesta biológica de las personas a ciertos estímulos externos, los medios más apropiados para estimular las percepciones sensoriales, las conductas asociadas a los diferentes estímulos, evaluar la efectividad de los medios usados y mejorar sus estrategias de marketing.

Randall Kevin (2016), establece que el neuromarketing consiste en la aplicación de las técnicas de investigación de las neurociencias a la investigación de marketing tradicional. A través de técnicas de medición de la actividad cerebral (como el EGG o la fMRI), las "respuestas" de los entrevistados a distintos estímulos (por ejemplo, anuncios publicitarios) son leídas directamente de su actividad cerebral. Las neurociencias permiten, por este método, averiguar que niveles de atención está prestando los sujetos analizados a un anuncio segundo por segundo y plano por plano. De este modo se pueden tomar decisiones como por ejemplo retirar un determinado plano del anuncio final o añadir una secuencia adicional. También pueden medirse otros muchos conceptos, como la activación del sujeto o su estado emocional cuando aparece el producto en pantalla.

\section{Resultados}

A continuación se muestran los resultados de la revisión de fuentes secundarias que contribuyan a la respuesta teórica y empírica del problema.

\subsection{Café y hogar}

Según comenta Alvarado Marsano (2013) cuando Clotaire Rapaille realizó una investigación en Estados Unidos por encargo de Procter \& Gamble, para descubrir cuál era el arquetipo cultural estadounidense de preferencias sobre el café, esta consultora descubrió que pese a los elevados niveles de consumo de los norteamericanos 
ellos no tenían preferencia por el sabor del café, entonces la decisión de compra no estaba relacionada con el sabor sino con otro factor. En este mismo estudio se identificó que en realidad los norteamericanos que consumen café en una mayoría asocian la experiencia con el aroma de café en las mañanas cuando vivían con sus padres, una asociación del aroma con la seguridad del hogar.

Entonces según Alvarado Marsano (2013), una emoción relacionada en el cerebro reptiliano y límbico, asocia el aroma con el hogar, que resulta ser el principal motivador de compra de café en los Estados Unidos. Empresas como Starbucks explotan muy bien esta asociación en sus campañas publicitarias.

\subsection{Neuromarketing visual}

En la ciudad de Cúcuta en Colombia, Avendaño Castro (2013), evidenció a través de la investigación realizada en el Centro Comercial Ventura Plaza de esa ciudad, que el uso de colores y luz en los establecimientos comerciales de ese centro, influyen sobre las decisiones de compra de los clientes, afirma sin embargo, que las prácticas de neuromarketing visual no necesariamente respondían a una planeación estratégica de las gerencias de los establecimientos.

Entre las conclusiones obtenidas del estudio realizado por Avendaño Castro (2013), mencionamos las siguientes:

El uso adecuado de los principios de la percepción (color e imagen fotográfica) producen una mayor atención de los consumidores al estimular la visión.

El color amarillo es mayoritariamente utilizado en las categorías de comida y libros. Este tono es el que genera mayor impacto en la retina del ojo humano y con esto, psicológicamente, induce a la acción.

El rojo es manejado en las categorías de comidas, diversión/ entretenimiento y electrodomésticos. Con este, se da un enfoque más emocional a las decisiones del consumidor y es punto clave para atraer al público joven.

En las categorías comerciales de textiles, comidas, diversión/entretenimiento, libros y electrodomésticos, poseen en su totalidad, una decoración acorde con los productos que ofrece al público, esto con ayuda de un espacio que hace cómodo el desplazamiento.

\subsection{El manejo de temáticas e imágenes influyen en la decisión de compra de los clientes}

ducen a sugerir que la estimulación a través del color, imagen y luz sobre la percepción sensorial visual, influye sobre la decisión de compra para satisfacer necesidades asociadas al cerebro límbico y reptiliano especialmente (hambre, vestir, confort, diversión, acción). Adicionalmente se evidencia, aunque no se explicita en la investigación, que en los establecimientos del centro comercial se aplican estrategias de segmentación (diferenciación demográfica) y de diferenciación entre tipos establecimientos caracterizados por el color, percepciones que también influyen en la conducta del consumidor.

\subsection{Neuromarketing y Publicidad}

Según la investigación realizada por León Carrascal (2014) a modo de hipótesis propone la siguiente interrogante: ¿Porque los profesionales en mercadeo y publicidad se apoyan del neuromarketing como una estrategia de comunicación?.

En el trabajo investigativo se propone que debido al número finito de compradores y la intensa competencia entre marcas, se deben considerar diferentes variables para influir en las decisiones de compra de las personas, la estimulación sensorial estrategicamente planificada hace que una marca sea más o menos atractiva que otra para los consumidores.

Al exponer una marca, el cerebro humano inicia con un reconocimiento de la misma, en donde relaciona lo que está viendo, escuchando o sintiendo y de esta manera se puede conseguir crear un posicionamiento de la marca. Es así como las personas tienen diferentes reacciones ante el mensaje enviado en algunos casos obteniendo resultados tanto positivos como negativos. (LEÓN CARRASCAL, 2014)

León Carrascal (2014) indica que al ser los sentidos, entendidos como percepciones sensoriales, el medio para que el cerebro perciba su entorno, la estimulación de ellos se convierte en un objetivo del marketing. La importancia de la publicidad esta en llegar adecuadamente a las percepciones de los consumidores de manera que se sientan interiormente atraidos, por ello los canales de comunicación y sus formas deben ser muy bien planificadas.

León Carrascal (2014) menciona que en el marketing y la publicidad, el neuromarketing se ha convertido en una estrategia de branding emocional, las personas cuando compran algo es por que quieren lo que ven, les agrada lo que huelen, sienten la comodidad, son atraidos por lo que escuchan o lo que degustan, son las emociones las que motivan la preferencia por los productos, de 
manera posterior recién racionalizan si el producto ha cumplido con sus expectativas. El cerebro humano esta en búsqueda permanente de su percepción de confort y satisfacción.

León Carrascal (2014) concluye que las empresas requieren del neuromarketing para impactar positivamente en el consumidor, desarrollando y publicitando productos acordes a los deseos de los mismos consumidores, cumpliendo el objetivo del marketing de ofrecer el mejor producto para satisfacer la necesidad.

Randall (2016), en su estudio, explica que el neuromarketing se puede aplicar para distintos propósitos de investigación. Aquí algunos ejemplos:

- Pretest de anuncios de televisión (Caso SONY Bravia) - Es quizá la aplicación más habitual del neuromarketing. Permite saber si el anuncio gusta o no gusta, seleccionar los planos estéticos, hacer pruebas con recursos creativos.

- Predicción de la viralidad de los anuncios (Caso SuperBowl) - Se ha utilizado para predecir que anuncios de la Super Bowl darían más que hablar en la red.

- Medición de Asociaciones de Marca - ¿Cómo de apropiado es un personaje para una marca? ¿Cómo de fuertes las asociaciones que el público tiene de esa marca?

\subsection{Cuasi Experimento relacionado con el Neuro- marketing}

Villalobos Contreras \& Soto de Clavero (2012) en el artículo Neuromarketing: Más Allá de la Teoría, Un Acercamiento Experimental al Cliente, sintetizan una investigación cuasi experimental realizada con un grupo de trece (13) personas. A continuación se describe el cuasi experimento desarrollado por ambas autoras:

En una Fase Inicial las autoras establecieron el comportamiento de los sujetos experimentales como reacción a estímulos orientados hacia sus sus deseos, reacción al manejo de comunicaciones y predisposición de apertura a la neurocomunicación, considerando además la influencia sobre sus decisiones de compra.

Para esta fase inicial, se sometió a los sujetos experimentales a procesos observados sobre isotipos, logotipos, marcas e imágenes de diferentes productos y servicios.

En el caso de los Isotipos, se presento 41 tipos, y debian responder la siguiente pregunta: ¿A qué empresa pertenece esta imagen o isotipo?
Para los Logotipos, 31 en total, debían responder la pregunta: Cuando observa este logotipo, ¿qué viene a su mente?

Para las Marcas, 37 presentadas, las pregunta a responder eran: ¿Qué sensación le producen estas marcas?, ¿cuál le inspira?, ¿cuál serías?, ¿con cuál dormirías?

En las Imágenes, se mostraron 80 , la pregunta realizada fue: De las siguientes imágenes, ¿qué emoción despierta en usted?

Las conclusiones llegadas de esta primera fase son, textualmente las siguientes:

[...], al comienzo los sujetos experimentales se encontraban tímidos y poco comunicativos, a pesar de que el Test de Inteligencia de RAVEN, logró despertar el sistema cerebral para alertar los procesos cognitivos y de pensamiento a los cuales se expusieron los sujetos. Casi al terminar la actividad cuando se introdujo el componente emocional a través de imágenes, fotos, situaciones, personajes, los sujetos experimentales consideraron expresar todo lo que sentían en ese momento, algunos más emotivos que otros con ciertas situaciones llegaron hasta casi llorar y recordar qué ocurría en sus vidas cuando vivieron esas imágenes.

Por tanto, se estableció que a nivel comunicacional, la imagen emocional vende y dispone al individuo para su fácil captación, sin embargo, el componente cerebral a nivel de pensamiento cognitivo hace funcionar más rápida y mejor la introducción de nuevos y viejos productos, marcas o servicios en las personas. (VILLALOBOS CONTRERAS; SOTO DE CLAVERO, 2012, p.)

En la Fase Experimental, Villalobos Contreras \& Soto de Clavero (2012), se propusieron demostrar que factores son los determinan el comportamiento de los sujetos experimentales como efecto de la neurocomunicación aplicada en el neuromercadeo. Esta fase fue dividida en seis partes:

Parte 1 - El comportamiento a través de la personalidad

Parte 2 - El Comportamiento durante la exposición a estímulos visuales, auditivos, olfativos, gustativos y táctiles

Parte 3 - Comportamiento basado en los deseos

Parte 4 -Tratamiento de las comunicaciones

Parte 5 - Aplicaciones de la neurocomunicación

Parte 6 - Toma de decisiones

A modo de reflexiones finales, Villalobos Contreras \& Soto de Clavero (2012), llegaron a las siguientes conclusiones del estudio cuasi experimental realizado: 
La estrategia de comunicaciones adecuadamente diseñada introduce conceptos nuevos en los procesos mentales de los consumidores para posicionar la imagen del producto y su asociación con la marca. Las personas asocian a través de sus sentidos sensaciones de placer confort en relación a un bien, lo que motiva emociones que inducen a la toma de decisiones de compra.

Aún cuando los estímulos son los mismos, la percepción es diferente para cada individuo. Los estímulos juegan un papel importante en las desiciones de los sujetos como consumidores. Las personas saben lo que quieren y como lo quieren, pero al experimentar estímulos en sus sentidos que son asociados a emociones de placer confort los hace más sensibles a determinadas empresas.

Finalmente Villalobos Contreras \& Soto de Clavero (2012), concluyen que el neuromarketing permite, a las empresas, identificar los principales factores que influyen sobre los sentidos de las personas, llegando a sí a influenciar a través de sus emociones, por asociación placer - confort, en sus decisiones de compra.

\subsection{Estrategias de mercadeo a partir del neuromar- keting}

El neuromarketing es una técnica del marketing que contribuye a entender el comportamiento del consumidor, a partir de la interpretación de las reacciones del cerebro a estimulaciones externas, la mejor comprensión de estos efectos permite a las empresas identificar estrategias de marketing que mejoren la posición de la empresa en el mercado.

Según Cortés Céspedes (2014) el neuromarketing apoya a identificar las conductas de los consumidores lo que hace más fácil diseñar estrategias de mercadeo, las relaciones entre estrategia de mercadeo en relación al neuromarketing se resumen a continuación:

- Segmentación - Permite identificar mejor los grupos con conductas y comportamientos similares.

- Posicionamiento - Contribuye a ubicar el producto en la mente del consumidor, generando recordación (top of mind), afecto (top of heart), posteriormente preferencia y lealtad (top of pocket).

- Targeting - Contribue a identificar con mayor eficacia el grupo objetivo para las campañas de mercadeo. El targeting junto a criterios de seg- grupos por similitudes de comportamiento, de manera que la comunicación, productos y servicios pueden ser diseñados para una mejor satisfacción de necesidades.

Diferenciación - Los criterios de diferenciación incluyen aspectos concientes e inconcientes, las emociones provocadas por ciertos productos, al ser caracterizadas pueden influir en las decisiones de las personas. Productos con características visibles similares pueden generar emociones muy diferentes si están asociadas a emociones diferentes.

En definitiva, el neuromarketing representa una oportunidad para generar estrategias de mercadeo desde el punto de vista del consumidor, así como desde el punto de vista del cliente si se trata de mercadear entre empresas, en este caso las estrategias se podrían llegar a desarrollar a través de técnicas del neuromanagement. (CORTÉS CÉSPEDES, 2014, p.)

Para Cortés Céspedes (2014), el neuromarketing al permitir interpretar mejor la conducta y comportamiento del consumidor aporta a mejorar las estrategias de marketing de las empresas.

\subsection{El mercado del neuromarketing}

En un mercado emergente como éste, el número de jugadores a nivel mundial es bastante pequeño. Despues de indagar la literatura respecto al tema se presenta una lista de algunas de las empresas más relacionadas con el neuromarketing o la medición biométrica. El país entre paréntesis indica procedencia, no ámbito de trabajo dado que la mayoría trabajan internacionalmente. (KLARIC, 2012).

- LAB (Polonia) (cerrada) Ahora son Human Mind \& Brain Applied Science Centre.

- Neurosense (Reino Unido)

- iMotions (Dinamarca)

- Neuro-Insight (Australia)

- NeuroFocus (EE.UU.)

- EmSense (EE.UU.) (cerrada)

- Buyology (EE.UU.)

- Sands Research (EE.UU.)

- Quantemo (EE.UU.)

- MindSign (EE.UU.)

- Neuromarketing (México)

- Lucid Systems (EE.UU.)

- Braidot Brain Decision Centre (Argentina)

- Eye Square (Berlín)

- Neurensics (Holanda) 
- Mindcode (EE.UU, México, Perú, Colombia y Bolivia)

- Loggicc (España)

- Neurobiomarketing (España)

- ICON Multimedia (España)

- BitBrain Technologies (España)

- InsideBrain (España)

\section{Discusión y conclusiones}

El objetivo de la investigación era identificar como las decisiones de compra son influenciadas por el neuromarketing, proponiendo para su logro la revisión de fuentes secundarias publicadas en español en el google académico en el periodo 2012 - 2016.

En una primera parte, se hizo una revisión teórica de las bases conceptuales que contribuyen a la formación del constructo neuromarketing:

- Cerebro triple y percepción sensorial cognitiva (base biológica)

- Necesidades como motivadores de compra

- Tecnologías de medición neurocientíficas

- Motivadores de las decisiones de compra

- Marketing y neuromarketing

De manera que se pueda comprender como los comportamientos del consumidor tienen base en conductas que se ubican de manera consciente o inconsciente en el cerebro humano; los aprendizajes actuales y más aún los ancestrales, resultan ser motivadores fuertes para la conducta humana.

En una segunda parte se ha realizado una búsqueda de evidencias documentales que permita evidenciar como estos motivadores alojados en el cerebro son influenciados por diferentes estimulaciones que terminan modificando las conductas de las personas influenciando sus comportamientos al momento de tomar una decisión de compra.

El análisis integrado de las bases teórico conceptuales y de las evidencias documentales, se registran algunos resultados que contribuyen a entender mejor como el neuromarketing influye en las decisiones de compra:

La conducta humana tiene una base biológica a partir de los aprendizajes alojados en el cerebro triple; estos aprendizajes influyen consciente o inconscientemente en las emociones aceptación-rechazó de las personas. p.e., en la mayoría de los norte americanos el aroma de café es asociado con una emoción aceptación, el recuerdo del hogar familiar. Este es un aprendizaje inconsciente del cerebro.
La caracterización descriptiva a partir de las tecnologías de la neurociencia, permite identificar las modificaciones en el cerebro ante estímulos sensoriales, de manera que se puede asociar el estímulo con una conducta de la persona, con lo que se tiene una mejor comprensión de las percepciones y expectativas de las personas.

El conocer mejor las percepciones y expectativas de las personas, da a las empresas la opción de tener mayor información para el diseño de productos y servicios que satisfagan mejor las necesidades de las personas.

La interpretación adecuada de las emociones asociadas a las conductas que influyen sobre el comportamiento del consumidor, hacen que las estrategias de marketing tengan mayor eficacia en la elección de los estimuladores sensoriales, influyendo en las preferencias de las personas.

Las personas compran si se sienten emocionalmente atraídas a un bien (producto o servicio), este comportamiento se observa aun al momento de elegir commodities.

Cuando los precios y productos son muy similares, la decisión de compra excluye estos atributos y las personas eligen aquel bien que asocian con una emoción positiva, entonces las empresas deberán identificar cuáles son esas emociones alojadas en el cerebro de la persona. El neuromarketing permite caracterizar estas emociones, de manera que las empresas puedan usar esta información para llegar al cliente.

Entonces el ¿Cómo?, está relacionado con el hecho que el neuromarketing es una técnica que permite conocer y pronosticar las conductas que influyen en los comportamientos de las personas al momento de tomar una decisión de compra.

El objetivo de la investigación fue logrado, no obstante, al ser las evidencias provenientes de fuentes secundarias y de realidades diferentes a las bolivianas, su validez debe ser probada con investigaciones experimentales.

\subsection{Limitaciones}

El estudio no permitió hacer una revisión literaria más profunda dado el escaso material disponible en revistas científicas respecto al tema.

Los artículos especializados en el tema y que corresponden al nivel ISI o JCR no estaban disponibles por razones de presupuesto para este documento.

La disponibilidad de información fue escasa para los autores del documento. 


\subsection{Líneas futuras de investigación}

Se considera imperante hacer estudios en empresas grandes bolivianas que tengan una orientación al marketing para conocer el grado de aplicabilidad de esta forma de hacer marketing.

También es importante considerar el hacer estudios de orden presupuestario para considerar la aplicación de la tecnología que a priori parece ser muy costosa en reemplazo de los métodos tradicionales para gestionar información precisa

Otra línea de investigación importante es la relación del neuromarketing con la gestión de la marca o el branding para establecer las posibilidades que el neuromarketing podría tener en la construcción del activo de marca.

\section{Referencias}

ALVARADO MARSANO, L. Neuromarketing, ciencia al servicio del conocimiento: sinergia e innovación. Repositório Académico UPC, v. 1, n. 2, p. 1-7, nov. 2013. Available in: <http://revistas.upc.edu.pe/index.php/sinergia/ article/view/128>. Access: 10 nov. 2016.

AVENDAÑO CASTRO, W. R. Un caso práctico para el análisis del neuromarketing visual en el Centro Comercial Ventura Plaza (Cúcuta, Colombia). Cuadernos de Administración, Facultad de Ciencias de la Administración, v. 29, n. 49, p. 17-27, 2013.

CAMACHO CASTELLANOS, J. C. El neuromarketing y su relación con las jerarquías de las necesidades de Abraham Maslow. Revista académica: contribuciones a la economia, ene./mar., 2016.

CANALES RONDA, P. Neuromarketing ¿El futuro ya está aquí? Ciencia, revista de investigación, v. 3, p. 2-11. 2013.
CORTÉS CÉSPEDES, A. I. El neuromarketing como metodología de investigación de mercados y herramienta de identificación en las decisiones de compra del consumidor. Colombia: Universidad Militar Nueva Granada, 2014.

DIEZ, M. Los tres cerebros, según MacLean. 2011. Available in: <http://martindiez.weebly.com/blog/los-tres-cerebros-segn-maclean>. Access: 10 nov. 2016.

KLARIC, Jurgen. Véndele a la mente, no a la gente. México: Biia International Publishing, 2015.

LEÓN CARRASCAL, X. C. El neuromarketing como nueva tendencia de la comunicación. España: Universidad Militar Nueva Granada, 2014.

MARTÍN CASTEJÓN, P. J.; LÁZARO PÉREZ, C. 2013. Neuromarketing: la última frontera de la comercialización. Gestión: revista de economía, v. 57, p. 14-18, 2013.

OSPINA GONZALEZ, L. A. Neuromarketing. Colombia: Universidad Militar Nueva Granada, 2014.

RANDALL, Kevin. Neuromarketing for branding. USA: Fast Company \& Inc Mansueto Ventures, 2016.

SILVA BUSTILLOS, R. Programación neurolingüística (PNL), neuromarketin y placebo. 2015. Available in: $\quad<$ http://dspace.ups.edu.ec/bitstream/123456789/11019/1/Programacion\%20Neurolinguistica\%20PNL\%20Neuromarketing\%20y\%20Placebo. pdf>. Access: 10 nov. 2016.

TALLADA CASANOVA, I. El neuormarketing hoy: realidad de su concepto e implantación. Madrid: Universidad Pontificia Comillas, 2015.

VIDAL BRAZALES, H. Estudios tradicionales en comportamiento del consumidor y nuevas tendências: el neuromarketing. Jaen: Universidad de Jaen, 2015.

VILLALOBOS CONTRERAS, M.; SOTO DE CLAVERO, A. I. Neuormarketing: más allá de la teoría, un acercamiento experimental al cliente. Revista Marketing Visionario, v. 1, n. 2, p. 23-43, 2012. 